\section{Tight Error Bounds for Nonuniform Signaling over AWGN Channels}

\author{
Hongyan Kuai, Fady Alajaji, Senior Member, IEEE, and \\ Glen Takahara, Member, IEEE
}

\begin{abstract}
We consider a Bonferroni-type lower bound due to Kounias on the probability of a finite union. The bound is expressed in terms of only the individual and pairwise event probabilities; however, it suffers from requiring an exponentially complex search for its direct implementation. We address this problem by presenting a practical algorithm for its evaluation. This bound is applied together with two other bounds, a recent lower bound (the KAT bound) and a greedy algorithm implementation of an upper bound due to Hunter, to examine the symbol error $\left(\boldsymbol{P}_{s}\right)$ and bit error $\left(\boldsymbol{P}_{b}\right)$ probabilities of an uncoded communication system used in conjunction with $M$-ary phase-shift keying (PSK)/quadrature amplitude (QAM) (PSK/QAM) modulations and maximum a posteriori (MAP) decoding over additive white Gaussian noise (AWGN) channels. It is shown that the bounds-which can be efficiently computed-provide an excellent estimate of the error probabilities over the entire range of the signal-tonoise ratio (SNR) $E_{b} / N_{0}$. The new algorithmic bound and the greedy bound are particularly impressive as they agree with the simulation results even during very severe channel conditions.
\end{abstract}

Index Terms-Additive white Gaussian noise (AWGN) channels, bit error rate (BER), lower bound, maximum a posteriori (MAP) and maximumlikelihood (ML) decoding, probability of a union, phase-shift keying (PSK) and quadrature amplitude (QAM) modulations, symbol error rate, upper bound.

\section{INTRODUCTION}

We consider a Bonferroni-type [12] lower bound on the probability $P\left(A_{1} \cup \cdots \cup A_{N}\right)$ of a finite union of $N$ events. This bound-which is due to Kounias [16] - is expressed in terms of only the individual ( $P\left(A_{i}\right)$ 's) and pairwise ( $P\left(A_{i} \cap A_{j}\right)$ 's) event probabilities. It is obtained as an optimal bound over a family of bounds which is indexed by the set of all subsets of $\{1,2, \ldots, N\}$; thus a direct search to determine the optimal bound requires a search over $2^{N}-1$ nonempty subsets, which is of exponential complexity. We have found in experiments in which various lower bounds were compared for small values of $N$ that the above bound was almost always the tightest. Thus it is of interest to have a practical implementation of this bound, or a near-optimal instance of it.

In this correspondence, we provide a novel stepwise algorithm which implements a suboptimal version of the Kounias lower bound; the complexity of this algorithm is empirically observed (in our applications) to be usually linear in $N$ and at worst $O\left(N^{2}\right)$. It is worth mentioning that this proposed algorithmic bound, along with a recent lower bound established by the authors in [19] (the KAT bound) and a greedy algorithm [1] implementation of an upper bound due to Hunter [14], provide new and computationally efficient techniques for error analysis of a variety of communication systems which have been traditionally handled using the union upper bound and variations of it.

One important communication system for which exact error probabilities are not available is when a nonuniformly distributed Bernoulli

Manuscript received May 28, 1999; revised May 16, 2000. This work was supported in part by the Natural Sciences and Engineering Research Council (NSERC) of Canada. The material in this correspondence was presented in part at the Eighth Communication Theory Mini-Conference, CTMC'99, Vancouver, BC, Canada, June 1999.

The authors are with Mathematics and Engineering, Department of Mathematics and Statistics, Queen's University, Kingston, ON, K7L 3N6 Canada.

Communicated by E. Soljanin, Associate Editor for Coding Techniques.

Publisher Item Identifier S 0018-9448(00)09680-2. source is transmitted via coherent $M$-ary phase-shift keying (PSK) and quadrature amplitude modulation (QAM) signaling with Gray code bit mapping over additive white Gaussian noise (AWGN) channels. We next investigate the application of the two algorithmic bounds (the stepwise algorithm lower bound and the greedy algorithm implementation of the Hunter upper bound) and the KAT lower bound to the probabilities of symbol error $P_{s}$ and bit error $P_{b}$ for this system. The justification for the nonuniformity assumption of the source stems from the fact that in many practical image and speech compression techniques, after some transformation, the transform coefficients are turned into bit streams (binary source). Due to the suboptimality of the compression scheme, the bit stream often exhibits a certain amount of redundancy [3], [6], [13], [29]. This embedded residual redundancy can be characterized by modeling the bit stream as an independent and identically distributed (i.i.d.) nonuniform (Bernoulli) process or as a Markov process [2], [3], [6], [17], [18].

When a nonuniform $M$-ary signal $s_{u}$ is transmitted over an AWGN channel, maximum a posteriori (MAP) decoding-which is optimal in the sense of minimizing the symbol error probability -is utilized at the receiver. Moreover, the more nonuniform the source is, the better is the system performance under the MAP decoding criterion ([2], [6], [13], see also Section IV). Under the MAP decoding rule, the symbol error probability can be written as

$$
P_{s}=\sum_{u=1}^{M} P\left(\epsilon \mid s_{u}\right) P\left(s_{u}\right)
$$

where $P\left(\epsilon \mid s_{u}\right)$ is the conditional probability of error given that $s_{u}$ was sent, and $P\left(s_{u}\right)$ is the probability of transmitting signal $s_{u}$. For most signal sets, an exact calculation of the right-hand side of the above equation is very difficult due to the complexity and nonsymmetrical structure of the decision regions. For this reason, upper and lower bounds on $P_{s}$ are of importance. Furthermore, calculation of the bit error probability $P_{b}$ is more complex than computing $P_{s}$ by an order of magnitude. In this work, the above three bounds are employed to estimate $P_{s}$ and $P_{b}$. The numerical computations of the bounds are shown to be excellent as both the stepwise lower bound and the greedy upper bound often coincide and agree with the simulation results even when the channel is very noisy.

In previous related work [7], Craig provided an elegant method for computing $P_{s}$ for two-dimensional $M$-ary signaling over AWGN channels. Although he implemented his technique exclusively for uniformly distributed (equally likely) signaling used with maximum-likelihood (ML) decoding, he briefly considered the problem of nonuniform signaling (with MAP decoding) and gave an expression for $P_{s}$ (cf. [7, eqs. (13) and (14)]). However, [7, eq. (13)] is not completely correct, as it assumes that each signal is inside its decision region which is not necessarily true for nonuniform signals with MAP decoding. Moreover, this expression is a sum of single integrals, the number and limits of which depend explicitly on the geometry of each decision region. In the case of uniform signaling, these regions are straightforward to determine and are symmetrically shaped. But for nonuniform signaling, these regions are nonsymmetric and are shaped differently for each signal. More specifically, each decision region is a (possibly infinite) polygon with up to $M-1$ sides determined by the intersection of $M-1$ half-planes, where the equation of each half-plane boundary is a function of the a priori signal probabilities and the channel noise variance. We have not encountered any efficient and systematic methods for numerically evaluating this expression in the literature. In this correspondence, we employ a different approach which does not require any 
knowledge of the geometry of the decision regions. With this method, we are also able to efficiently estimate $P_{b}$ for nonuniform signaling.

In [4], [26], and [10], Craig's method was extensively generalized to provide a unified framework for the determination of $P_{s}$ for various uniform modulation schemes over generalized fading channels. In other work, the computation of $P_{b}$ for uniform $M$-ary PSK and QAM signaling over AWGN channels was investigated in [21], [15], and [20]. In an approach similar in spirit to our correspondence, Séguin [23] employed an inequality by de Caen [9] to derive a lower bound on the probability of codeword error of uniform $M$-ary signals derived from binary linear codes and sent over AWGN channels. We note that de Caen's bound is weaker than the KAT lower bound [19]; thus the results in [23] can be tightened using the bounds presented in this correspondence.

The rest of this correspondence is organized as follows. In Section II, the three general bounds on the probability of a finite union of events are described in detail. In Section III, the problem of estimating the symbol and bit error probabilities of nonuniform $M$-ary signals transmitted via PSK/QAM modulation over AWGN channels is investigated. Numerical results and discussions are presented in Section IV. Finally, conclusions are stated in Section V.

\section{BOUNDS ON THE PROBABILITY OF A UNION}

Consider a finite family of events $A_{1}, A_{2}, \ldots, A_{N}$ in an arbitrary probability space $(\Omega, P)$, where $N$ is a fixed positive integer. In this section, we present a practical algorithmic lower bound to $P\left(\bigcup_{i=1}^{N} A_{i}\right)$ and briefly describe an algorithmic implementation of an upper bound due to Hunter [14] and a recent lower bound established by the authors in [19].

\section{A. Stepwise Algorithm Lower Bound}

In this subsection, we present an algorithmic lower bound on the probability of a finite union. The bound is a suboptimal instance of the following bound by Kounias [16], which is generally infeasible to determine exactly

$$
P\left(\bigcup_{i=1}^{N} A_{i}\right) \geq \max _{\mathcal{J}}\left\{\sum_{i \in \mathcal{J}} P\left(A_{i}\right)-\sum_{\substack{i, j \in \mathcal{J} \\ i<j}} P\left(A_{i} \cap A_{j}\right)\right\}
$$

where $\mathcal{J} \subseteq \mathcal{S} \triangleq\{1,2, \ldots, N\}$. We note that the above maximization requires a search over $2^{N}-1$ subsets; this results in a search complexity that increases exponentially with $N$. We significantly reduce this complexity by proposing a suboptimal algorithm whose search complexity is empirically observed (in our applications) to be usually linear in $N$ and at worst $O\left(N^{2}\right)$. It employs a stepwise searching technique which is commonly used in statistics to search for the optimal subset of predictors in regression problems involving a large number of candidates (see, for example, [28]). In the statistical applications, the objective function is usually based on a goodness of fit measure, such as the Akaike Information Criterion (AIC) [28]. In our problem, the objective is to maximize the term in braces on the right-hand side (RHS) of (1).

Let $M(\mathcal{J})$ denote the term in braces on the RHS of (1) for subset $\mathcal{J} \subseteq \mathcal{S}$. The basic idea of the algorithm is to (iteratively) construct a subset $\mathcal{J}$ such that both $M(\mathcal{J} \cup\{i\})$ and $M(\mathcal{J} \backslash\{j\})$ are not greater than $M(\mathcal{J})$ for any index $i \notin \mathcal{J}$ on index $j \in \mathcal{J}$. Specifically, the algorithm is as follows.

\section{Stepwise Algorithm:}

- Step 0: Set $\mathcal{J}_{1}=\emptyset$ and $\mathcal{J}_{2}=\mathcal{S}$. Set $M\left(\mathcal{J}_{1}\right)=0$ and compute $M\left(\mathcal{J}_{2}\right)$
- Step 1:

- $\quad$ la: Augment $\mathcal{J}_{1}$ by adding to it (if possible) the index $i \in \mathcal{S} \backslash \mathcal{J}_{1}$ such that the term in braces on the RHS of (1) is maximized and $M\left(\mathcal{J}_{1} \cup\{i\}\right)>M\left(\mathcal{J}_{1}\right)$.

- $\quad 1 b$ : Shrink $\mathcal{J}_{2}$ by deleting from it (if possible) the index $j \in \mathcal{J}_{2}$ such that the term in braces on the RHS of (1) is maximized and $M\left(\mathcal{J}_{2} \backslash\{j\}\right)>M\left(\mathcal{J}_{2}\right)$.

- Step 2: Repeat Step 1 until the term in braces on the RHS of (1) can no longer be improved in either Steps 1a or $1 b$.

- Step 3:

- 3a: Shrink $\mathcal{J}_{1}$ by deleting (if possible) the index $j \in \mathcal{J}_{1}$ such that the term in braces on the RHS of (1) is maximized and $M\left(\mathcal{J}_{1} \backslash\{j\}\right)>M\left(\mathcal{J}_{1}\right)$.

- 3b: Augment $\mathcal{J}_{2}$ by adding to it (if possible) the index $i \in \mathcal{S} \backslash \mathcal{J}_{2}$ such that the term in braces on the RHS of (1) is maximized and $M\left(\mathcal{J}_{2} \cup\{i\}\right)>M\left(\mathcal{J}_{2}\right)$.

- Step 4: Repeat Step 3 until the term in braces on the RHS of (1) can no longer be improved in either Steps 3a or 3b.

- Step 5: Repeat Steps 1-4 until the metric $M$ can no longer be improved. Output the stepwise lower bound as

$$
\max \left\{M\left(\mathcal{J}_{1}\right), M\left(\mathcal{J}_{2}\right)\right\}
$$

The above algorithm actually implements the stepwise procedure twice, once starting with the empty set and once starting with the set of all indexes $\mathcal{S}$, and chooses the better of the two results. In our applications we observed that, starting with either set $(\emptyset$ or $\mathcal{S})$, the algorithm goes through a process of successively deleting or adding indexes from the current subset at most once, resulting in a linear search complexity.

\section{B. Greedy Algorithm Upper Bound}

We herein describe an algorithmic upper bound on the probability of a union. As in the case for the lower bound, this upper bound is expressed in terms of the individual and pairwise error probabilities. We first present a family of upper bounds due to Hunter.

Theorem 1 ([14], [12]): Let $A_{1}, A_{2}, \ldots, A_{N}$ be $N$ sets, where $N \geq 3$. Then

$$
P\left(\bigcup_{i=1}^{N} A_{i}\right) \leq \sum_{i=1}^{N} P\left(A_{i}\right)-\sum_{(i, j) \in T_{0}} P\left(A_{i} \cap A_{j}\right)
$$

where $T_{0}$ is any tree spanning the $N$ indices of the sets $A_{1}$, $A_{2}, \ldots, A_{N}$ and $(i, j)$ is an edge in $T_{0}$.

Since $T_{0}$ is arbitrary, the tightest bound in this family is obtained as follows:

$$
P\left(\bigcup_{i=1}^{N} A_{i}\right) \leq \sum_{i=1}^{N} P\left(A_{i}\right)-\max _{T_{0} \in \mathcal{T}} \sum_{(i, j) \in T_{0}} P\left(A_{i} \cap A_{j}\right)
$$

where $\mathcal{T}$ is the set of all spanning trees of the $N$ indexes. This set $\mathcal{T}$ has $N^{N-2}$ elements [27]; thus a direct search to compute (3) has an exponential complexity. However, the problem of finding the optimal tree can be formulated as that of determining the maximal spanning tree of a completely connected weighted graph $G$ with nodes corresponding to the event indexes and edge weights corresponding to the pairwise event probabilities. Viewed in this way, the Greedy Algorithm [27], [1] (also known as Kruskal's algorithm) for obtaining a minimal spanning tree for a weighted graph can be applied to construct the optimal spanning tree $T_{0}$ for (3). The algorithm is described as follows.

Greedy Algorithm: Consider a (fully) connected graph $G$ with $N$ vertices and $\left(\begin{array}{c}N \\ 2\end{array}\right)$ edges $(i, j)$ of weights $P\left(A_{i} \cap A_{j}\right)$. Construct a set of edges $T_{0}$ as follows. 
- Step 0: $T_{0}=\emptyset$.

- Step 1: Add to $T_{0}$ the edge with maximum weight.

- Step 2: From the remaining edges, add to $T_{0}$ the edge with maximum weight subject to the constraint that $T_{0}$ remain cycle-free.

- Step 3: Repeat Step 2 until $T_{0}$ contains $N-1$ edges.

The complexity of this algorithm is theoretically at worst $O\left(N^{3}\right)$ [1]. However, as for the stepwise bound, we observed in our applications a search complexity that was usually linear. We also note that there exists an improved implementation of this algorithm which has theoretically at worst $O\left(N^{2}\right)$ search complexity [1].

\section{KAT Lower Bound}

In [19], Kuai, Alajaji, and Takahara prove the following bound.

Theorem 2 ([19]): KAT lower bound

$$
\begin{array}{r}
P\left(\bigcup_{i=1}^{N} A_{i}\right) \geq \sum_{i=1}^{N}\left(\frac{\theta_{i} P\left(A_{i}\right)^{2}}{\sum_{j=1}^{N} P\left(A_{i} \cap A_{j}\right)+\left(1-\theta_{i}\right) P\left(A_{i}\right)}\right. \\
\left.+\frac{\left(1-\theta_{i}\right) P\left(A_{i}\right)^{2}}{\sum_{j=1}^{N} P\left(A_{i} \cap A_{j}\right)-\theta_{i} P\left(A_{i}\right)}\right)
\end{array}
$$

where

and

$$
\begin{aligned}
\theta_{i} & \triangleq \frac{\beta_{i}}{\alpha_{i}}-\left\lfloor\frac{\beta_{i}}{\alpha_{i}}\right\rfloor \\
\alpha_{i} & \triangleq P\left(A_{i}\right)
\end{aligned}
$$

$$
\beta_{i} \triangleq \sum_{j: j \neq i} P\left(A_{i} \cap A_{j}\right)
$$

In [19], it was also demonstrated that de Caen's lower bound [9] and the Dawson-Sankoff lower bound [8] are special cases of the above lower bound. When $\theta_{i}=0$ for all $i$, the KAT lower bound reduces to de Caen's bound; when the ratio $\beta_{i} / \alpha_{i}$ is constant, it reduces to Dawson-Sankoff's bound. Furthermore, it is shown in [19] that the KAT bound is always sharper than these two bounds.

\section{NONUNIForm Signaling Over AWGN ChanNElS}

We apply the bounds to estimate the symbol error probability $\left(P_{s}\right)$ and the bit error probability $\left(P_{b}\right)$ of nonuniform $M$-PSK or $M$-QAM modulated AWGN channels. The problem formulation is as follows.

We consider a nonuniform i.i.d. binary source $\left\{X_{i}\right\}$, with distribution $P\{X=0\}=p$, that is transmitted via $M$-PSK or $M$-QAM modulation over an AWGN channel with single-sided power spectral density $N_{0}$. The source stream is grouped in blocks of $\log _{2} M$ bits which are each subsequently mapped to a modulation signal for transmission over the channel (we assume that $M$ is a power of 2). At the receiver, optimal MAP decoding is performed in estimating the transmitted $M$-ary signal. More specifically, if one of $M$ signals $s_{1}, s_{2}, \ldots, s_{M}$ is sent, then the MAP decoder declares that $s_{k}$ was sent if, for $i=1,2, \ldots, M$ and $i \neq k$, the MAP metric of $s_{k}$ is bigger than the metric of $s_{i}$; i.e.,

$$
P\left(s_{k} \mid r\right) \geq P\left(s_{i} \mid r\right)
$$

where $r=s_{u}+n$ is the received signal and $n$ is Gaussian distributed noise with zero-mean and covariance matrix $\left(N_{0} / 2\right) I_{2}$, where $I_{2}$ is the $2 \times 2$ identity matrix.

\section{A. Average Symbol Error Rate}

The probability of symbol decoding error $P_{s}$ is

$$
P_{s}=\sum_{u=1}^{M} P\left(\epsilon \mid s_{u}\right) P\left(s_{u}\right)=\sum_{u=1}^{M} P\left(\bigcup_{i \neq u} \epsilon_{i u}\right) P\left(s_{u}\right)
$$

where $P\left(\epsilon \mid s_{u}\right)$ is the conditional probability of error given that $s_{u}$ was sent, and $\epsilon_{i u}$ represents the event that $s_{i}$ has a higher MAP metric than $s_{u}$ given that $s_{u}$ was sent. Therefore,

$$
P\left(\epsilon_{i u}\right)=\operatorname{Pr}\left\{p\left(r \mid s_{i}\right) p\left(s_{i}\right) \geq p\left(r \mid s_{u}\right) p\left(s_{u}\right)\right\}
$$

and

$$
\begin{array}{r}
P\left(\epsilon_{i u} \cap \epsilon_{j u}\right)=\operatorname{Pr}\left\{p\left(r \mid s_{i}\right) p\left(s_{i}\right) \geq p\left(r \mid s_{u}\right) p\left(s_{u}\right),\right. \\
\left.p\left(r \mid s_{j}\right) p\left(s_{j}\right) \geq p\left(r \mid s_{u}\right) p\left(s_{u}\right)\right\} .
\end{array}
$$

The RHS of (6) can be easily reduced to

$$
\operatorname{Pr}\left\{N_{0} \ln p\left(s_{i}\right)-\left\|n+s_{u}-s_{i}\right\|^{2} \geq N_{0} \ln p\left(s_{u}\right)-\|n\|^{2}\right\}
$$

which is

$$
\operatorname{Pr}\left\{\frac{\sqrt{2}\left\langle n, s_{i}-s_{u}\right\rangle}{\sqrt{N_{0}}\left\|s_{i}-s_{u}\right\|} \geq \frac{\left\|s_{i}-s_{u}\right\|}{\sqrt{2 N_{0}}}+\frac{N_{0} \ln \frac{p\left(s_{u}\right)}{p\left(s_{i}\right)}}{\sqrt{2 N_{0}}\left\|s_{i}-s_{u}\right\|}\right\} .
$$

Notice that

$$
X_{i}=\frac{\sqrt{2}}{\sqrt{N_{0}}\left\|s_{i}-s_{u}\right\|}\left\langle n, s_{i}-s_{u}\right\rangle
$$

is Gaussian with 0 -mean and unit variance. Then

$$
P\left(\epsilon_{i u}\right)=Q\left(\frac{d_{i u}}{\sqrt{2 N_{0}}}+\frac{\sqrt{2 N_{0}} \ln P\left(s_{u}\right) / P\left(s_{i}\right)}{2 d_{i u}}\right)
$$

and similarly

$$
\begin{array}{r}
P\left(\epsilon_{i u} \cap \epsilon_{j u}\right)=\Psi\left(\rho_{i j u}, \frac{d_{i u}}{\sqrt{2 N_{0}}}+\frac{\sqrt{2 N_{0}} \ln P\left(s_{u}\right) / P\left(s_{i}\right)}{2 d_{i u}},\right. \\
\left.\frac{d_{j u}}{\sqrt{2 N_{0}}}+\frac{\sqrt{2 N_{0}} \ln P\left(s_{u}\right) / P\left(s_{j}\right)}{2 d_{j u}}\right)
\end{array}
$$

where

$$
\begin{aligned}
d_{i u} & =\left\|s_{i}-s_{u}\right\|, \\
\rho_{i j u} & =\frac{\left\langle s_{i}-s_{u}, s_{j}-s_{u}\right\rangle}{\left\|s_{i}-s_{u}\right\| \cdot\left\|s_{j}-s_{u}\right\|} \\
Q(x) & =\frac{1}{\sqrt{2 \pi}} \int_{x}^{\infty} \exp \left(-y^{2} / 2\right) d y \\
\Psi\left(\rho_{i j u}, a, b\right) & =\frac{1}{2 \pi \sqrt{1-\rho_{i j u}^{2}}} \int_{a}^{\infty} \int_{b}^{\infty} e^{-\frac{\left(x^{2}-2 \rho_{i j u} x y+y^{2}\right)}{2\left(1-\rho_{i j u}^{2}\right)}} d x d y
\end{aligned}
$$

where $\|\cdot\|$ is the Euclidean norm and $\langle\cdot, \cdot\rangle$ denotes the usual dot product.

If we apply the algorithms in Sections II-A and II-B and Theorem 2 to $P\left(\bigcup_{i \neq u} \epsilon_{i u}\right)$ and substitute in (5), we obtain two lower bounds and one upper bound on $P_{s}$ in terms of $P\left(\epsilon_{i u}\right), P\left(\epsilon_{i u} \cap \epsilon_{j u}\right)$, and $P\left(s_{u}\right)$.

\section{B. Average Bit Error Rate (BER)}

In many cases, the bit error probability is a more useful performance measure. Under the MAP decoding criterion, the bit error probability $P_{b}$ can be written as

$$
P_{b}=\sum_{u=1}^{M} P_{b}(u) P\left(s_{u}\right)
$$


where

$$
\begin{aligned}
P_{b}(u) & =\frac{1}{\log _{2} M} E\left(\# \text { of bit errors } \mid s_{u} \text { is sent }\right) \\
& =\frac{1}{\log _{2} M} \sum_{j=1}^{M} d\left(c_{j}, c_{u}\right) A_{j / u}
\end{aligned}
$$

and

$$
\begin{aligned}
A_{j / u} & =P\left(s_{j} \text { is decoded } \mid s_{u} \text { is sent }\right) \\
& =1-P\left(\bigcup_{i \neq j}\left\{P\left(s_{i} \mid r\right) \geq P\left(s_{j} \mid r\right) \mid s_{u} \text { is sent }\right\}\right) \\
& =1-P\left(\bigcup_{i \neq j} \epsilon_{i j u}\right)
\end{aligned}
$$

where $u=1, \ldots, M, c_{j}$ and $c_{u}$ are the bit assignments for signals $s_{j}$ and $s_{u}$, respectively, $d\left(c_{j}, c_{u}\right)$ is the Hamming distance between $c_{j}$ and $c_{u}$, and $\epsilon_{i j u}$ represents the event that symbol $s_{i}$ has a higher metric than symbol $s_{j}$ given that symbol $s_{u}$ was sent. As in the case for the symbol error probability, $P\left(\epsilon_{i j u}\right)$ and $P\left(\epsilon_{i j u} \cap \epsilon_{k j u}\right)$ can be expressed in terms of the $Q(\cdot)$ and $\Psi(\cdot)$ functions, respectively. More precisely, we obtain that

$$
\begin{aligned}
P\left(\epsilon_{i j u}\right)= & \operatorname{Pr}\left\{p\left(r \mid s_{i}\right) p\left(s_{i}\right) \geq p\left(r \mid s_{j}\right) p\left(s_{j}\right) \mid s_{u} \text { is sent }\right\} \\
= & \operatorname{Pr}\left\{N_{0} \ln p\left(s_{i}\right)-\left\|n+s_{u}-s_{i}\right\|^{2}\right. \\
& \left.\geq N_{0} \ln p\left(s_{j}\right)-\left\|n+s_{u}-s_{j}\right\|^{2}\right\} \\
= & \operatorname{Pr}\left\{\frac{\sqrt{2}\left\langle n, s_{i}-s_{j}\right\rangle}{\sqrt{N_{0}}\left\|s_{i}-s_{j}\right\|} \geq \frac{N_{0} \ln \frac{p\left(s_{j}\right)}{p\left(s_{i}\right)}}{\sqrt{2 N_{0}}\left\|s_{i}-s_{j}\right\|}\right. \\
& \left.\quad+\frac{\left\|s_{i}-s_{u}\right\|^{2}}{\sqrt{2 N_{0}}\left\|s_{i}-s_{j}\right\|}-\frac{\left\|s_{j}-s_{u}\right\|^{2}}{\sqrt{2 N_{0}}\left\|s_{i}-s_{j}\right\|}\right\} \\
= & \left(\frac{N_{0} \ln \frac{p\left(s_{j}\right)}{p\left(s_{i}\right)}}{\sqrt{2 N_{0}}\left\|s_{i}-s_{j}\right\|}+\frac{\left\|s_{i}-s_{u}\right\|^{2}}{\sqrt{2 N_{0}}\left\|s_{i}-s_{j}\right\|}\right. \\
& \left.-\frac{\left\|s_{j}-s_{u}\right\|^{2}}{\sqrt{2 N_{0}}\left\|s_{i}-s_{j}\right\|}\right)
\end{aligned}
$$

and

$$
\begin{aligned}
& P\left(\epsilon_{i j u} \cap \epsilon_{k j u}\right)=\operatorname{Pr}\left\{p\left(r \mid s_{i}\right) p\left(s_{i}\right) \geq p\left(r \mid s_{j}\right) p\left(s_{j}\right),\right. \\
& \left.p\left(r \mid s_{k}\right) p\left(s_{k}\right) \geq p\left(r \mid s_{j}\right) p\left(s_{j}\right)\right\} \\
& =\Psi\left(\rho_{i k j}, \frac{N_{0} \ln \frac{p\left(s_{j}\right)}{p\left(s_{i}\right)}}{\sqrt{2 N_{0}}\left\|s_{i}-s_{j}\right\|}\right. \\
& +\frac{\left\|s_{i}-s_{u}\right\|^{2}}{\sqrt{2 N_{0}}\left\|s_{i}-s_{j}\right\|}-\frac{\left\|s_{j}-s_{u}\right\|^{2}}{\sqrt{2 N_{0}}\left\|s_{i}-s_{j}\right\|}, \\
& \frac{N_{0} \ln \frac{p\left(s_{j}\right)}{p\left(s_{k}\right)}}{\sqrt{2 N_{0}}\left\|s_{k}-s_{j}\right\|}+\frac{\left\|s_{k}-s_{u}\right\|^{2}}{\sqrt{2 N_{0}}\left\|s_{k}-s_{j}\right\|} \\
& \left.-\frac{\left\|s_{j}-s_{u}\right\|^{2}}{\sqrt{2 N_{0}}\left\|s_{k}-s_{j}\right\|}\right)
\end{aligned}
$$

where

$$
\rho_{i k j}=\frac{\left\langle s_{i}-s_{j}, s_{k}-s_{j}\right\rangle}{\left\|s_{i}-s_{j}\right\| \cdot\left\|s_{k}-s_{j}\right\|}
$$

Finally, applying the algorithms in Sections II-A and II-B and Theorem 2 to $P\left(\bigcup_{i \neq j} \epsilon_{i j u}\right)$ in (9) yields two upper bounds and one lower bound on the bit error probability $P_{b}$.

\section{NUMERICAL RESULTS AND DisCUSSION}

In this section, we apply the results of Sections II and III to present bounds for the probability of symbol error $P_{s}$ and bit error $P_{b}$ for nonuniform signals with 8-PSK, 16-PSK, 32-PSK, and 16-QAM modulation (using Gray mapping) over AWGN channels. We consider $p=$ 0.5 and 0.9 for the probability that an input source bit is zero. The results for symbol and bit error are displayed in terms of the signal-tonoise ratio (SNR) $E_{b} / N_{0}$, where $E_{b}$ is the energy per information bit. The SNR ranges considered are all from $\mathrm{SNR}=-4$ to $\mathrm{SNR}=h$, with $h$ ranging from 4 to 14 , so the focus is on noisy channels for which the usual union bound performs poorly. For evaluation of the bounds, we also provide simulation results for $p=0.9$. We provide the union upper bound in the plots for symbol-error rate (SER). We do not show the KAT-based upper bound in the bit-eror rate (BER) plots, as its performance in relation to the stepwise bound is similar to its performance in the SER plots.

We first consider the case $p=0.5$ for which MAP estimation is equivalent to ML estimation. We note that for $p=0.5$ with $M$-PSK or $M$-QAM modulation, exact methods are available for $P_{s}$ (e.g., [22]) and $P_{b}$ ([21], [15], [20]), so that bounds are unnecessary. However, for $M$-PSK modulation ( $M=8,16,32$ ) we have found the lower and upper bounds for both symbol error $\left(P_{s}\right)$ and bit error $\left(P_{b}\right)$ derived in Sections III-A and III-B, based on the stepwise and greedy bounds from Sections II-A and II-B, to coincide, and agree with the exact values for $P_{s}$ and $P_{b}$. Thus either of these bounds may simply be viewed as alternative methods for computing the exact symbol- or bit-error rates in these cases. For the 16-QAM case, we plot the symbol and bit errors in Figs. 1 and 2. Though not exact for this case, the stepwise and greedy-based bounds are excellent over the entire range of SNR values. We also show in these figures the simulation results for a highly nonuniform source with $p=0.9$. These curves, when compared with the plots for $p=0.5$, illustrate the fact that the system performance is considerably improved via increased nonuniformity and the use of MAP decoding.

As mentioned, exact methods are available in the case of uniform signals. The use of these bounds provides equally precise probabilities in a practical sense. Their added value is that the same code can be used to estimate performance for nonuniform signals, which are considered in the following.

For the case of nonuniform signaling, we have found no published results for efficiently calculating $P_{s}$ or $P_{b}$ exactly; thus bounds are very useful. In Figs. 3 to 6, the bounds for the symbol- and bit-error rates are plotted for $p=0.9$. In all cases, the stepwise and greedy bounds provide excellent results, being very close to exact over the entire range of SNR values. The union upper bound for SER, as expected, does not perform well at lower SNR, particularly for the 32-PSK case in Fig. 5.

The KAT lower bound for the SER provides very good results, though not as good as the stepwise lower bound for SER, and the accuracy seems to degrade as $M$ increases in the case of $M$-PSK modulation. It should be remembered, however, that the KAT lower bound is a single formula which, given the single and pairwise event error probabilities, does not involve a search over a large class of bounds, and so may be more practical for applications which involve a very large number of events in the union of interest; for example, in analyzing code or BERs in complex coded systems (cf. [23]).

For the applications considered in this correspondence, all bounds were practical to compute. For the SER curves, for a given value of SNR, and including the time to calculate all the single and pairwise event probabilities, the combined computing time to calculate all bounds (KAT, stepwise, greedy, and union) was less than a second for 8-PSK and 1 or $2 \mathrm{~s}$ for 32-PSK, on a computer with a 266-MHz MMX Pentium chip running Linux. For the $P_{b}$ plots (described below), the 


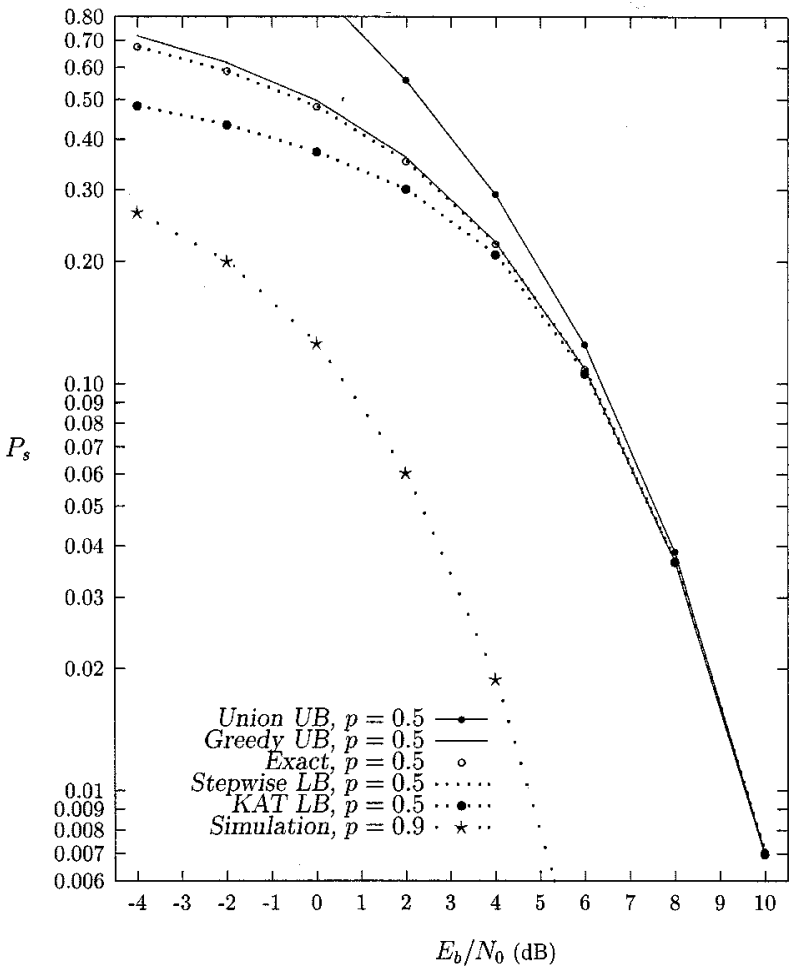

Fig. 1. Symbol error rate $P_{s}$ for 16-QAM.

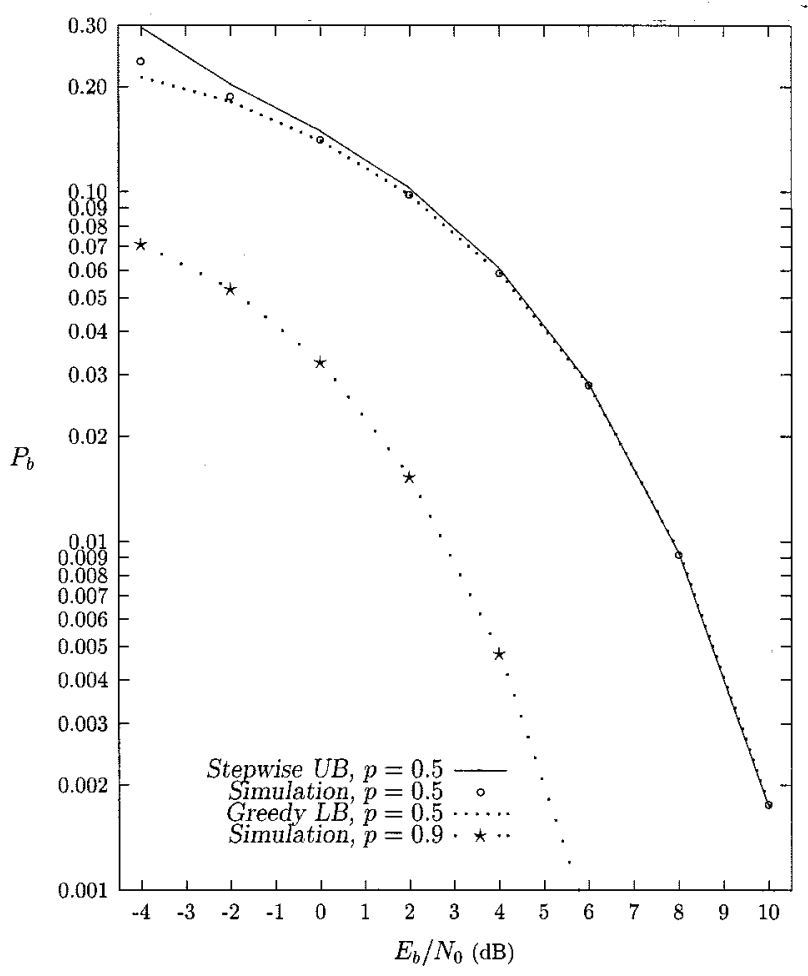

Fig. 2. BER $P_{b}$ for 16-QAM.

corresponding times for just the KAT upper bound (computed but not shown in this correspondence) were less than a second even for 32-PSK, while the times for the stepwise or greedy bounds were about $1 \mathrm{~s}$ for 8 -PSK and up to $2 \mathrm{~min}$ for 32-PSK.

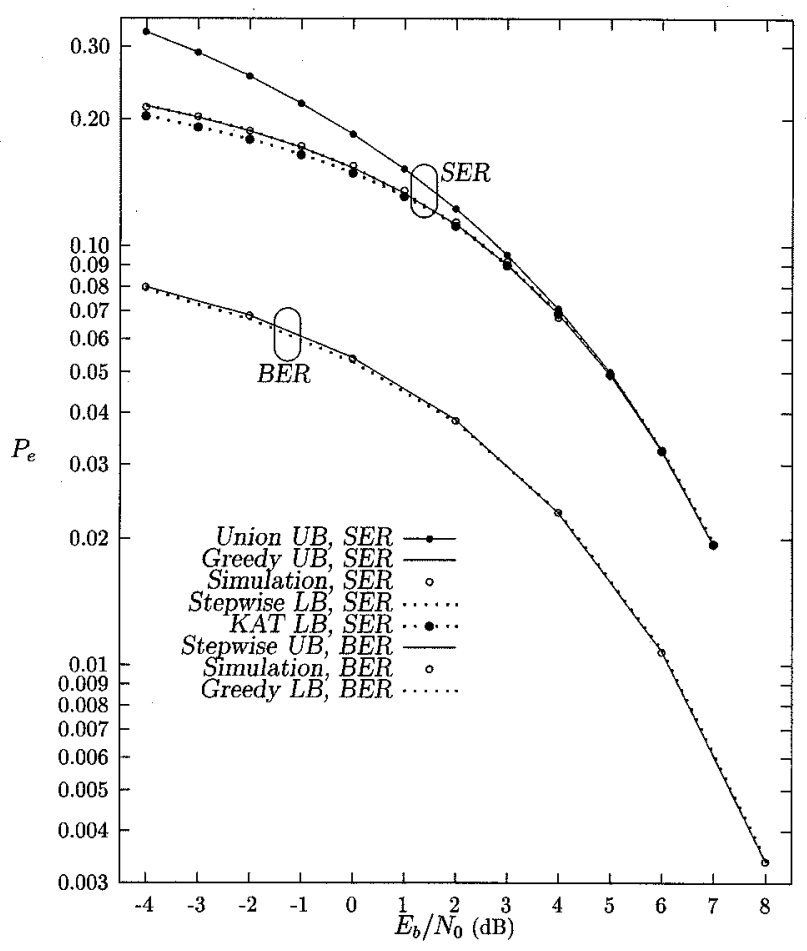

Fig. 3. Symbol error rate $P_{s}$ and BER $P_{b}$ for 8-PSK, $p=0.9$.

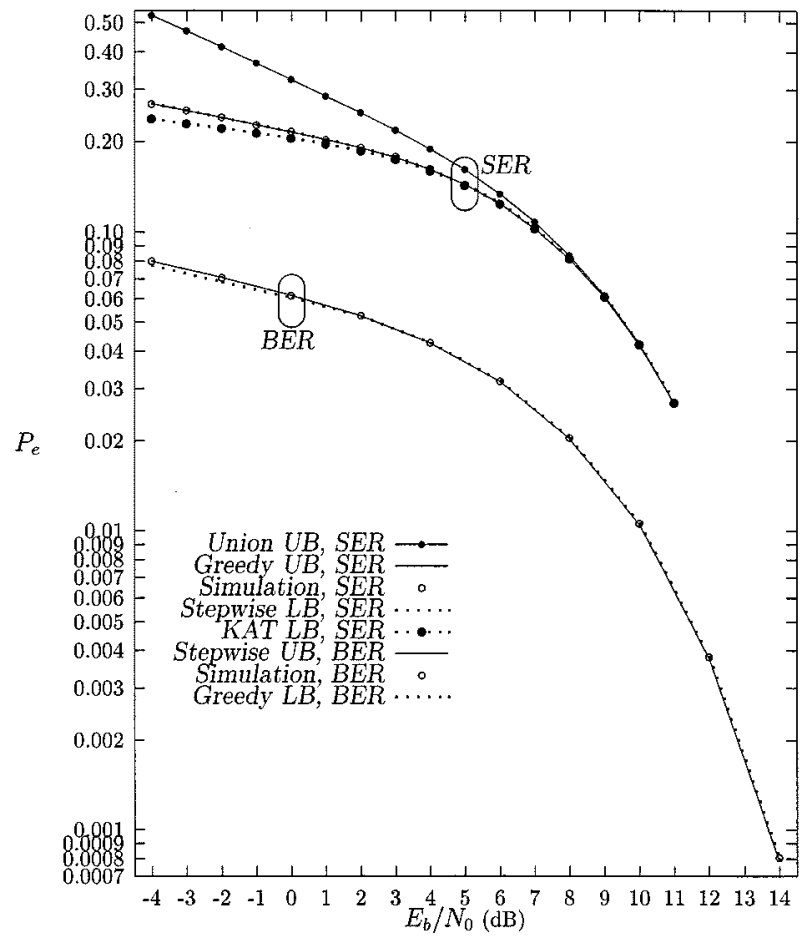

Fig. 4. Symbol error rate $P_{s}$ and BER $P_{b}$ for 16-PSK, $p=0.9$.

We next mention the algorithms used to compute the $Q$ and $\psi$ functions of Sections III-A and III-B. For $Q(\cdot)$, we adopted the algorithm developed by Beaulieu [5], which takes only a few lines of code and which we have found to be very efficient and accurate. For the $\Psi(\cdot)$ function, we adopted an algorithm for computing bivariate normal tail probabilities written in Fortran by Donnelly [11] (see also [24]). We 


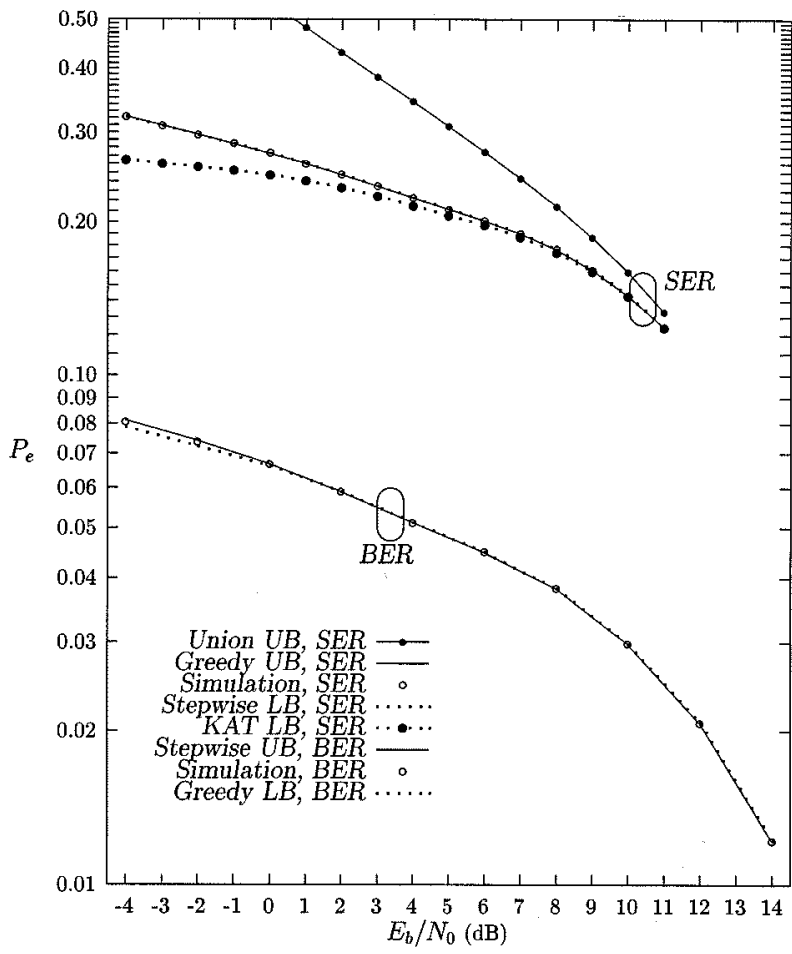

Fig. 5. Symbol error rate $P_{s}$ and BER $P_{b}$ for 32-PSK, $p=0.9$.

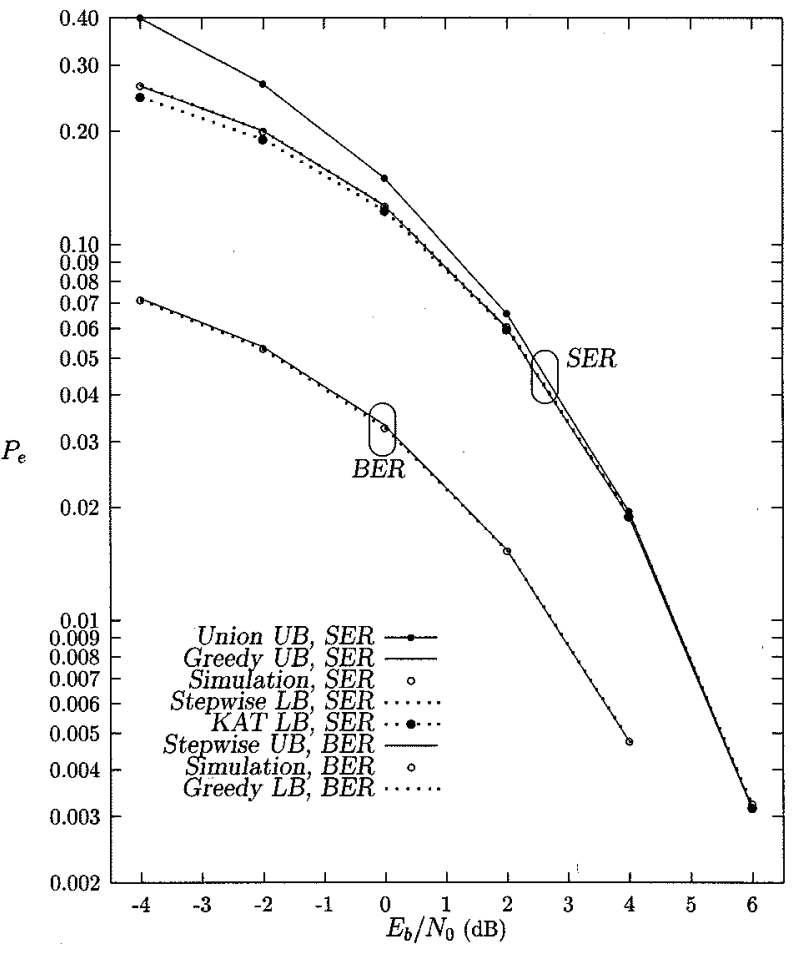

Fig. 6. Symbol error rate $P_{s}$ and BER $P_{b}$ for 16-QAM, $p=0.9$.

note that naive computation of the double integral in the $\Psi(\cdot)$ function by simple numerical integration rules results in a very inefficient algorithm that takes over a day of computing to produce, for example, the values for the SER curves for 16-PSK in Fig. 4. For nonnegative lower limits, Simon and Divsalar [25] (see also [26]) provide an alternate representation of the $\Psi$ function as the sum of two single in- tegrals over finite ranges. For uniform signaling, the lower limits in (7) or (11) are both nonnegative, but for nonuniform signaling, one or both of these limits can be negative, in which case the $\Psi$ function can be expressed as a linear combination of four instances of the $\Psi$ function with nonnegative lower limits. However, with the application of Simpson's composite numerical integration rule, this method proved much less efficient for a given accuracy as compared with Donnelly's algorithm, which is based on rational function approximations. Thus the latter method was adopted.

Additional results for the computation of the SER and BER bounds when $p=0.7$ were obtained. The accuracy of the stepwise and greedy bounds were again shown to be excellent. However, we do not include them for the sake of brevity.

\section{CONCLUSION}

A stepwise algorithmic lower bound on the probability of a finite union, which is expressed in terms of only the individual and pairwise intersection event probabilities, was presented. This bound is a practical implementation of a bound that is otherwise exponentially complex to evaluate.

This bound along with two other bounds, a recent lower bound due to the authors (the KAT bound) and a greedy algorithm implementation of an upper bound due to Hunter, were applied for the estimation of the symbol and bit error probabilities of nonuniform $M$-PSK/QAM signaling over very noisy AWGN communication channels used in conjunction with MAP decoding. The stepwise and greedy bounds provided excellent and often exact estimates of $P_{s}$ and $P_{b}$ for all examples considered. The KAT bound was also shown to provide very good bounds for most cases considered. The main advantage of the KAT bound is its computational simplicity, which can be an advantage when one wishes to calculate the probability of the union of a very large number of events, or the probability of a small or moderate union many times.

It should be noted that the bounds we have presented are very general and can be applied to any problem involving calculation of the probability of events which can be expressed as a finite union of events whose individual and pairwise intersection probabilities can be efficiently computed. Future work may include the application of these bounds to Rayleigh fading channels and the analysis of channel coded communication systems with nonuniform inputs using MAP decoding.

\section{REFERENCES}

[1] R. K. Ahuja, T. L. Magnanti, and J. B. Orlin, Network Flows. Englewood Cliffs, NJ: Prentice Hall, 1993.

[2] F. Alajaji, N. Phamdo, N. Farvardin, and T. Fuja, "Detection of binary Markov sources over channels with additive Markov noise," IEEE Trans. Inform. Theory, vol. 42, pp. 230-239, Jan. 1996.

[3] F. Alajaji, N. Phamdo, and T. Fuja, "Channel codes that exploit the residual redundancy in CELP-encoded speech," IEEE Trans. Speech Audio Processing, vol. 4, pp. 325-336, Sept. 1996.

[4] M.-S. Alouini and A. Goldsmith, "A unified approach for calculating error rates of linearly modulated signals over generalized fading channels," IEEE Trans. Commun., vol. 47, pp. 1324-1334, Sept. 1999.

[5] N. C. Beaulieu, "A simple series for personal computer computation of the error function (.)," IEEE Trans. Commun., vol. 37, pp. 989-991, Sept. 1989.

[6] P. Burlina and F. Alajaji, "An error resilient scheme for image transmission over noisy channels with memory," IEEE Trans. Image Processing, vol. 7, pp. 593-600, Apr. 1998.

[7] J. W. Craig, "A new simple and exact result for calculating the probability of error for two-dimensional signal constellations," in Proc. IEEE Military Commun. Conf., McLean, VA, Oct. 1991, pp. 571-575.

[8] D. A. Dawson and D. Sankoff, "An inequality for probabilities," Proc. Amer. Math. Soc., vol. 18, pp. 504-507, 1967.

[9] D. de Caen, "A lower bound on the probability of a union," Discr. Math., vol. 169, pp. 217-220, 1997. 
[10] X. Dong, N. C. Beaulieu, and P. H. Wittke, "Error probabilities of twodimensional $M$-ary signaling in fading," IEEE Trans. Commun., vol. 47 pp. 352-355, Mar. 1999.

[11] T. G. Donnelly, "Bivariate normal distribution," Commun. ACM, vol. 16, p. 636, 1973. Algorithm 462.

[12] J. Galambos and I. Simonelli, Bonferroni-Type Inequalities with Applications. New York: Springer-Verlag, 1996.

[13] J. Hagenauer, "Joint source and channel coding for broadcast applications," in Audio and Video Digital Radio Broadcasting Systems and Techniques. Amsterdam, The Netherlands: Elsevier, 1994.

[14] D. Hunter, "An upper bound for the probability of a union," J. Appl. Probab., vol. 13, pp. 597-603, 1976.

[15] M. I. Irshid and I. S. Salous, "Bit error probability for coherent $M$-ary PSK systems," IEEE Trans. Commun., vol. 39, pp. 349-352, Mar. 1991.

[16] E. G. Kounias, "Bounds on the probability of a union, with applications," Ann. Math. Statist., vol. 39, no. 6, pp. 2154-2158, 1968.

[17] J. Kroll and N. Phamdo, "Analysis and design of trellis codes optimized for a binary symmetric markov source with maximum a posteriori detection," IEEE Trans. Inform. Theory, vol. 44, pp. 2977-2987, Nov. 1998.

[18] _ "Source-channel optimized trellis codes for bitonal image transmission over AWGN channels," IEEE Trans. Image Processing, vol. 8, pp. 899-912, July 1999.

[19] H. Kuai, F. Alajaji, and G. Takahara, "A lower bound on the probability of a finite union of events," Discr. Math., vol. 215, pp. 147-158, Mar. 2000.

[20] P. J. Lee, "Computation of bit error rate of coherent $M$-ary PSK with gray code bit mapping," IEEE Trans. Commun., vol. 34, pp. 488-491, May 1986.

[21] J. Lu, K. B. Letaief, J. C.-I. Chuang, and M. L. Liou, " $M$-PSK and $M$-QAM BER computation using signal-space concepts," IEEE Trans. Commun., vol. 47, pp. 181-184, Feb. 1999.

[22] J. G. Proakis, Digital Communications. New York: McGraw-Hill, 1995.

[23] G. E. Séguin, "A lower bound on the error probability for signals in white Gaussian noise," IEEE Trans. Inform. Theory, vol. 44, pp. 3168-3175, Nov. 1998.

[24] M. J. Schervish, "Multivariate normal probabilities with error bound," Appl. Statist., vol. 33, no. 1, pp. 81-94, 1984. Algorithm AS 195.

[25] M. K. Simon and D. Divsalar, "Some new twists to problems involving the Gaussian probability integral," IEEE Trans. Commun., vol. 46, pp. 200-210, Feb. 1998.

[26] M. K. Simon and M. S. Alouini, "A unified approach to the performance analysis of digital communication over generalized fading channels," Proc. IEEE, vol. 86, pp. 1860-1877, Sept. 1998.

[27] J. H. van Lint and R. M. Wilson, A Course in Combinatorics. Cambridge, MA: MIT Press, 1992.

[28] W. N. Venables and B. D. Ripley, Modern Applied Statistics with S-Plus, 2nd ed. New York: Springer-Verlag, 1997.

[29] W. Xu, J. Hagenauer, and J. Hollmann, "Joint source-channel decoding using the residual redundancy in compressed images," in Proc. Int. Conf. Communications, Dallas, TX, June 1996.

\section{Optimum Asymptotic Multiuser Efficiency of Randomly Spread CDMA}

David N. C. Tse, Member, IEEE, and Sergio Verdú, Fellow, IEEE

Abstract-This correspondence analyzes the high signal-to-noise ratio (SNR) performance of optimum multiuser detectors for synchronous direct-sequence spread spectrum with random spreading in an additive white Gaussian noise channel. Under very general conditions on the received powers, we show that the optimum asymptotic efficiency of a $K$-user system with spreading gain $N$ converges to 1 almost surely as $K \rightarrow \infty$, and $K / N$ is kept equal to an arbitrary nonzero constant. Therefore, the asymptotic behavior of the minimum bit error rate is equivalent to that of a single-user system.

Index Terms-Asymptotic efficiency, CDMA, multiuser detection, optimum multiuser detection, spread spectrum.

\section{INTRODUCTION}

This correspondence is concerned with the analysis of the capabilities of optimum multiuser detection for the basic synchronous CDMA multiple-access $K$-user channel [1]

$$
y(t)=\sum_{k=1}^{K} A_{k} b_{k} s_{k}(t)+\sigma n(t)
$$

where $A_{k} \in(0, \infty), b_{k} \in\{-1,1\}$ and $s_{k}$ are the received amplitudes, data, and unit-energy signature waveform of the $k$ th user, respectively, and $n(t)$ is additive white Gaussian noise.

Uncoded bit error rate has received much attention as a performance measure of multiuser detectors. Of particular interest is the asymptotic multiuser efficiency which characterizes the performance loss (in effective signal-to-noise ratio (SNR)) as the background noise vanishes. If a particular receiver achieves bit-error rate $\mathrm{P}_{k}(\sigma)$ in the presence of multiple-access interference and additive white Gaussian noise with power spectral level equal to $\sigma^{2}$, then the asymptotic multiuser efficiency is given by [1]

$$
\eta_{k}=\frac{2}{A_{k}^{2}} \lim _{\sigma \rightarrow 0} \sigma^{2} \log 1 / \mathrm{P}_{k}(\sigma) .
$$

The analysis of the error probability of optimum multiuser detectors for arbitrary signature waveforms and received powers goes back more than fifteen years [2]-[4]. Denote the diagonal matrix of received amplitudes $\boldsymbol{A}=\operatorname{diag}\left\{A_{1}, \ldots, A_{K}\right\}$, and the normalized crosscorrelation matrix by $\boldsymbol{R}$, with entries $\rho_{i j}=\left\langle s_{i}, s_{j}\right\rangle$. The optimum asymptotic multiuser efficiency is equal to [1]

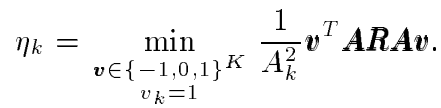

Manuscript received November 3, 1999; revised May 29, 2000. This work was supported by the NSF under CAREER Award and the U.S. Army Research Office under Grant DAAH04-96-1-0379. The material in this correspondence was presented in part at the Information Theory Workshop, Santa Fe, NM, February 1999

D. N. C. Tse is with the Department of Electrical Engineering and Computer Sciences, University of California, Berkeley CA 94720 USA (e-mail: dtse@eecs. berkeley.edu).

$\mathrm{S}$. Verdú is with the Department of Electrical Engineering, Princeton University, Princeton, NJ 08540 USA (e-mail: verdu@ @rinceton.edu)

Communicated by U. Madhow, Associate Editor for Detection and Estimation.

Publisher Item Identifier S 0018-9448(00)09658-9. 